\section{Charles Mock Introduction}

\author{
John G. Hunter
}

(C) Société Internationale de Chirurgie 2011

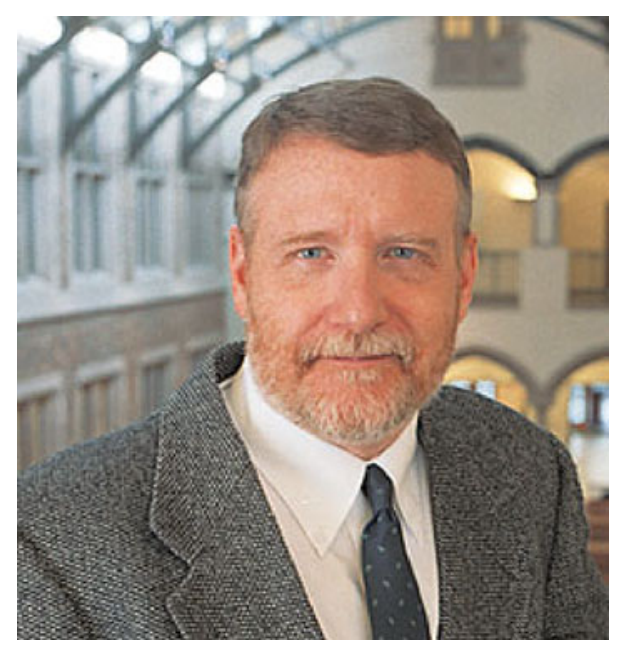

It gives me a great deal of pleasure to introduce a new WJS associate editor, Charles Mock MD, PhD. Global health and the support of surgical research, education, and clinical care in low- and middle-income countries are primary missions of the World Journal of Surgery. Charlie Mock will give us additional expertise in this area, to provide the highest-quality editorial review and content development.

Dr. Mock grew up in the United States and has recently returned from Geneva to resume his position as Professor of Surgery at the University of Washington in Seattle. He has spent his career directly involved in providing surgical care in "under-resourced" settings from the rural United States to rural Africa. Equally important, Dr. Mock has created and supported innovative international policy for the care of the injured and burned patient while working at the World Health Organization in Geneva. He has been a leader in several the international societies addressing the surgical needs of patients in low- and middle-income countries, including our own IATSIC. Dr. Mock has been a frequent contributor and reviewer for the WJS over the past 5 years. A journal is only as strong as its editorial board, its reviewers, and its authors. We are thrilled that such a talented educator, surgeon, and health policy researcher has joined our senior editorial ranks. Charles Mock will make the World Journal of Surgery stronger.
J. G. Hunter $(\bowtie)$

Department of Surgery, Oregon Health \& Science University, 3181 SW Sam Jackson Park Road, Portland, OR 97239, USA e-mail: hunterj@ohsu.edu 